\title{
PROJECT-ECHO: REACHING BEST PRACTICES IN GYNECOLOGIC ONCOLOGY THROUGH INTERNATIONAL VIDEOCONFERENCING WITH CASE MANAGEMENT AND DIDACTICS
}

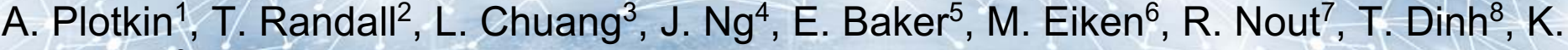 Schmeler ${ }^{9}$.
${ }^{1}$ University of Toronto, Pathology, Toronto, Canada. ${ }^{2}$ Massachusetts General Hospital, Gynecologic Oncology, Boston, USA. ${ }^{3}$ Western Connecticut Health Network, Obstetrics and Gynecology, Danbury, USA. ${ }^{4}$ National University Cancer Institute, Gynecologic Oncology, Singapore, Singapore. ${ }^{5} \mathrm{MD}$ Anderson Cancer Center, Cancer Prevention and Population Sciences, Houston, USA. ${ }^{6}$ International Gynecologic Cancer Society, IGCS, Chicago, USA. ${ }^{2}$ Leiden University Medical Center, Radiation Oncology, Leiden, The Netherlands.
${ }^{8}$ Mayo Clinic, Obstetrics and Gynecology, Jacksonville, USA. ${ }^{9}$ MD Anderson Cancer Center, Gynecologic Oncology, Houston, USA.

\section{Objectives}

The IGCS has expanded to include all regions of the world in the past three years, collaborating with many national societies and launching the Global Curriculum for gynecologic oncology fellowship training in LMICs. With this growth there has been great enthusiasm to learn from new colleagues. The extension for Community Healthcare Outcomes (Project $\mathrm{ECHO}$ ) telementoring model has been known to improve patient outcomes in low resource areas through sharing of best practices in specialty care.

\section{Methods}

The IGCS, in collaboration with MD Anderson Cancer Center, set up a Project ECHO platform in 2017 to connect members through monthly virtual tumor boards with planned didactic teaching sessions. Local sites present cases with relevant history, imaging and pathology. Multi-institutional groups of IGCS experts discuss best practices and make recommendations on management.

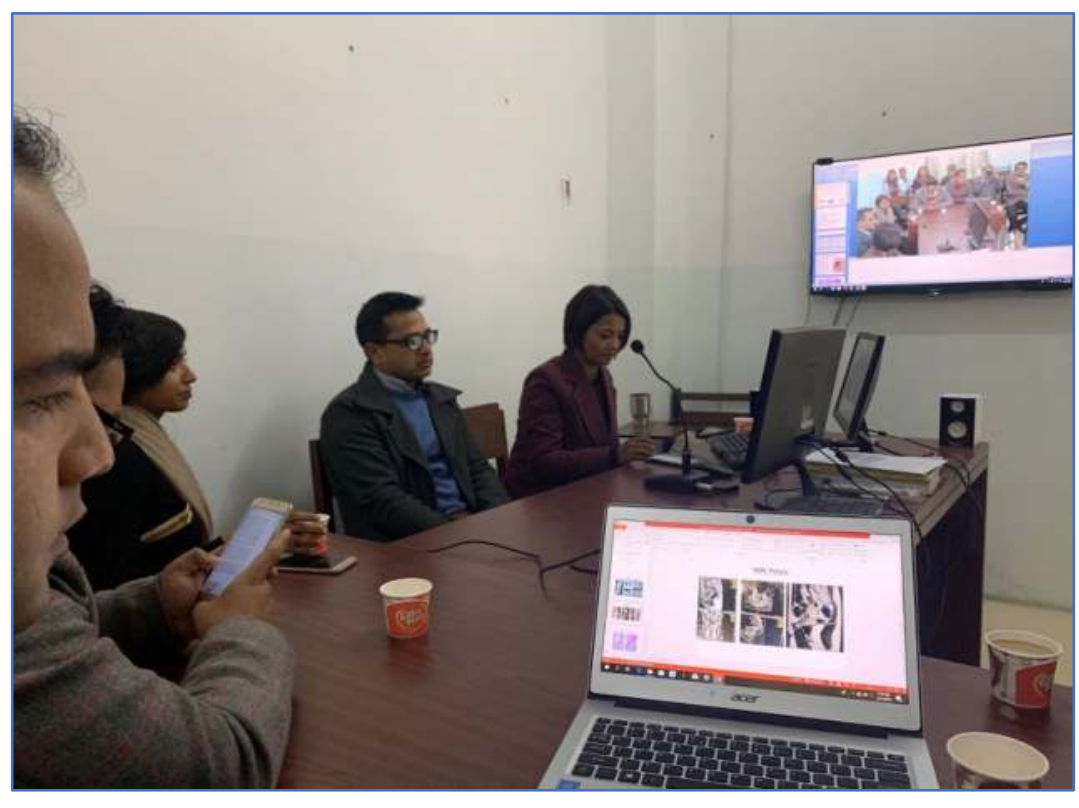

Results

The IGCS currently has 13 host sites participating in Project ECHO. Ten of these are Global Curriculum sites, while three are sites with established gynecologic oncology training seeking greater exchange of ideas in best practices. In 2018, 8 sites held 47 total Project ECHO sessions. Verbal and written feedback has been highly positive.

\section{Conclusions}

Remote telementoring through Project $\mathrm{ECHO}$ videoconferences is feasible and acceptable, and highly valued by participants, across widely disparate settings. 